\title{
Lebensmittelkontrolle und Verbraucherinformation im digitalen Zeitalter
}

\author{
Georg A. Schreiber
}

Published online: 5 November 2013

(C) Bundesamt für Verbraucherschutz und Lebensmittelsicherheit (BVL) 2013

Wie, Sie kaufen immer noch Ihre Lebensmittel im Supermarkt? Hetzen alle paar Tage durch dieselben Regalreihen und stapeln die gleichen Produkte im Einkaufswagen, warten genervt an der Kasse und wuchten die Einkaufstüten, das Flüssigwaschmittel, die Kiste Mineralwasser und den $10 \mathrm{~kg}$ schweren Sack Katzenstreu in den viel zu kleinen Kofferraum und dann wieder raus und in die Wohnung? Das ist gestern! Fragen Sie mal Ihr Digital-Native-Kid.

In Seoul hat Tesco schon im August 2011 einen virtuellen Supermarkt in einer U-Bahnstation installiert: großflächige Abbildungen von gefüllten Regalen. Die Produkte mit QR-Code, die via Smartphone in den Warenkorb befördert werden, während man auf die U-Bahn wartet, elektronische Bezahlung und Lieferung am Abend in die Wohnung. Fertig. Das ist heute! Und die Einkaufsliste wird beim nächsten Mal aufgerufen, editiert und abgeschickt - schon wieder fertig.

Einkaufen via Internet? Für Tickets selbstverständlich. Aber Lebensmittel? Das ist neu für uns. Im Vergleich zu Franzosen, Engländern und Schweizern hängen Deutsche weit hinterher. In Deutschland geben Verbraucherinnen und Verbraucher an, die Ware selbst aussuchen zu wollen. Anfassen zu können und Beratung wären wichtig. Tatsächlich? Oder hängen wir einer Idylle vom Tante-Emma-Laden mit individueller Beratung hinterher, den wir durch

Dr. G. A. Schreiber ( $\bowtie)$

Bundesamt für Verbraucherschutz und

Lebensmittelsicherheit, Mauerstr. 39-42, 10117 Berlin,

Germany

e-mail: georg.schreiber@bvl.bund.de unser Rennen in die Geiz-ist-geil-Tempel längst verdrängt haben?

Deutsche stehen Neuerungen per se skeptisch gegenüber. Warum sollte das beim Lebensmitteleinkauf im Internet anders sein. Z.Z. steht auch die hohe Supermarktdichte dem Durchstarten entgegen. Das ist in Frankreich und England anders, wo Verbraucherinnen und Verbraucher längere Wege zum nächsten Supermarkt haben. Des Weiteren die geringe Gewinnmarge der deutschen Ketten, die zusätzliche Investitionen in Transportsysteme für den letzten Kilometer zum Kunden schwierig machen. Mittlerweile setzt sich aber die Erkenntnis durch, dass ein zu spätes Aufspringen auf den Zug das Ende sein könnte. Also springen die Großen auf und bieten ihren Kunden in vielfältiger Weise die OnlineBestellung und das bequeme Abholen oder die Lieferung am Abend nach Hause an. Die Kleinen bieten diesen Service längst.

Als Online-Kunden drängeln heute schon die gestressten Berufstätigen, die eher nach als vor 20 Uhr aus dem Büro kommen, die Mütter und Väter, die Großeinkäufe erledigen müssen, die Digital Natives, die zunehmend die Elternhäuser verlassen und eigene Haushalte gründen, und die Silver Surfer, die durch den Online-Kauf und insbesondere die Lieferung nach Hause sich eine längere Unabhängigkeit erkaufen, ${ }^{1}$ das Angebot und den Lieferservice zu verbessern. Die letzt genannten Gruppen werden schnell wachsen und man muss kein Wahrsager sein:

\footnotetext{
${ }^{1}$ Miriam Linder, Carsten Rennhak, Lebensmittel-Onlinehandel in Deutschland, Reutlinger Diskussionsbeiträge zu Marketing \& Management, ESB Business School, Hochschule Reutlingen, Nr. 2012-4.
} 
In 10 Jahren werden viele der rechteckigen Kästen, die in den letzten zwei Jahrzehnten in unseren Städten und an den Dorfrändern wie Pilze aus dem Boden geschossen sind, verschwunden sein - hoffentlich rückgebaut. Man muss ihnen keine Träne nachweinen. Die Entwicklung wird sie überflüssig gemacht haben. Bleiben oder zurückkommen werden hoffentlich die kleinen Läden für das individuelle Freizeitshoppen oder für solche Produkte, die man lieber selbst abholt, wie das Frühstücksbrötchen.

Diese Entwicklung wird nicht nur den Handel verändern. Es sind Transportsysteme gefragt, die ein Höchstmaß an Kundenservice bieten, also intelligente Lösungen für den letzten Kilometer. Auch die Steuerung der Timeslots für Lieferungen über Smartphone, verschiedene Temperaturbereiche in den Lieferfahrzeugen, Rücknahme von Leergut und unbürokratische Erstattung von beschädigter oder nicht gewünschter Ware sind nur einige der weiteren Themen. Daneben werden die Kontrollbehörden mit völlig neuen Problemen konfrontiert. Muss die amtliche Lebensmittelüberwachung sich um den Zustand der Ware, wie sie beim Kunden ankommt, kümmern? Oder reicht die Überprüfung der Transportsysteme aus? „Sind wir überhaupt zuständig?“, eine beliebte Frage in Amtsstuben, wird häufig gestellt werden.

Und wie findet die Behörde den Lebensmittelhändler, der vorgibt in „Kleindettelau“ zu sitzen, tatsächlich aber von den Bahamas aus versucht, deutsche Verbraucherinnen und Verbraucher hinters Licht $\mathrm{zu}$ führen? Darauf haben die Behörden bereits eine Antwort, auch wenn nicht jeder Händler, der illegale Absichten verfolgt, gefunden werden kann: die Gemeinsame Zentralstelle der Länder im Bundesamt für Verbraucherschutz und Lebensmittelsicherheit „Kontrolle der im Internet gehandelten Erzeugnisse des LFGB und Tabakerzeugnisse“,2 etwas kürzer G@ZIELT. In effizienter Art wird durch G@ZIELTmit nur sechs Mitarbeiterinnen und Mitarbeitern das Angebot an Lebensmitteln, Futtermitteln, Kosmetika, Bedarfsgegenständen und Tabakprodukten sowie deren Anbieter für ganz Deutschland recherchiert. Wesentlich ist die Zusammenarbeit mit vier Siegelgebern, die die Kriterien der Initiative D21 erfüllen. ${ }^{3}$ Die Siegel auf einer Website, auf der Lebensmittel angeboten werden, sagen der Verbraucherin und dem Verbraucher, dass der Anbieter bzw. sein Betrieb von der zuständigen Behörde überwacht wird. Damit bietet das Online-

\footnotetext{
${ }^{2}$ http://www.bvl.bund.de/internethandel

${ }^{3}$ http://www.internet-guetesiegel.de
}

Unternehmen das gleiche Sicherheitsniveau wie das entsprechende konventionelle Unternehmen. Es ist die Herausforderung für Behörden, Siegelgeber und Online-Lebensmittelunternehmen, den Verbraucherinnen und Verbrauchern die Bedeutung der Siegel nahe zu bringen und sie darüber zu informieren. Erst, wenn die Verbraucherinnen und Verbraucher von der Sicherheit der online angebotenen Lebensmittel überzeugt sind, bzw. wissen, wie sie Risiken minimieren können, wird der breite Durchbruch des OnlineHandels im Lebensmittelbereich stattfinden. Es würde schließlich auch niemand Online-Tickets kaufen, wenn nicht sicher wäre, dass man damit ins Konzert kommen oder mit der Bahn fahren kann.

Information der Verbraucherinnen und Verbraucher wird im digitalen Zeitalter immer wichtiger. Zum einen fordern die Verbraucherinnen und Verbraucher selbst eine bessere Information. Sie wollen wissen, wie die Produkte und deren Zutaten hergestellt wurden und wo sie herkommen. In einer Zeit, in der höchstens noch Fachleute die Produktionsbedingungen und Herkünfte überblicken, eine verständliche, aber oft nur schwer zu erfüllende Forderung. Zum anderen können Behörden und Hersteller das Internet nutzen, um Verbraucherinnen und Verbraucher zu schützen bzw. über Gefahren und Risiken besser zu informieren. Das Portal www.lebensmittelwarnung.de ist ein Beispiel wie Behörden zusammenarbeiten können, um Verbraucherinnen und Verbraucher effektiv zu informieren, und dabei keinerlei Berührungsängste mit modernen Kommunikationstechniken oder -diensten wie Twitter und RSS-Feed zeigen.

Die diesjährige Summer School der Hochschule für angewandte Wissenschaften Hamburg hat sich dieser Thematik, „Information der Verbraucherinnen und Verbraucher", gewidmet. In dieser Ausgabe des JVL sind Kurzzusammenfassungen der verschiedenen RednerBeiträge zu finden. Es werden u.a. die Auswirkungen des überarbeiteten Verbraucherinformationsgesetzes aus Sicht der Wirtschaft und der Behörden beleuchtet. Erfüllt es nun endlich den Zweck, der Verbraucherin und dem Verbraucher zu ermöglichen, bei Behörden Information abzurufen, oder muss es in Verbraucherorganisationsinformationsgesetz umbenannt werden? Es wird beleuchtet, ob das Portal www. lebensmittelklarheit.de, das Verbraucherinnen und Verbrauchern die Möglichkeit gibt, ihre subjektive Meinung über Produkte zu äußern, tatsächlich ein Erfolg ist. Aus Sicht der Verbraucherorganisationen sicherlich, immerhin wurde eine Vielzahl von Produkten aufgrund der Beschwerden von den Herstellern verändert. Die Wirtschaft dagegen hat das Portal immer als Pranger verteufelt, anscheinend ohne darüber 
nachzudenken, dass die Einschätzung und Meinung von Verbraucherinnen und Verbrauchern über bestimmte Produkte nicht nur Denunziation bedeuten muss, sondern auch einen wertvollen Erkenntnisgewinn darstellen kann. Und auch die Diskussion zum Smiley für Lebensmittelbetriebe fehlt nicht. Das Thema wird seit Jahren von Politikern und Behörden mit deutscher Gründlichkeit hin und her diskutiert, während andere Staaten den Smiley einfach einführen - ohne größere Schwierigkeiten. Einem Verbraucher die Information über das Ergebnis der letzten amtlichen Kontrolle seines Lieblingsrestaurants zu verheimlichen, ist von gestern! Das werden auch die Wirtschaftsministerinnen und -minister irgendwann einsehen müssen. Über Google-Maps die Smileys der Restaurants meiner Straße abrufen zu können, damit ich unter Einbeziehung weiterer Parameter, wie Geschmack und Zufriedenheit beim letzten Besuch, Speisekarte und Preis eine mündige Entscheidung treffen kann, ist seit Jahren gängige Praxis in Dänemark.
Das digitale Zeitalter stellt Behörden vor viele Herausforderungen, gibt aber auch eine Fülle von Möglichkeiten. Mit dem Online-Angebot und -Verkauf von Produkten, die bisher nur im Laden an der Ecke oder im Supermarkt zu haben waren, sehen sich die Behörden herausgefordert, ihre Kontrollprozeduren auf die virtuelle Ebene zu heben. Daneben eröffnet das Internet und seine vielfältigen Dienste Möglichkeiten, Verbraucherinnen und Verbraucher viel besser als bisher zu erreichen und auch deren Meinung und Bedürfnisse viel leichter erfassen zu können als im vorherigen Zeitalter. Die Behörden sind mit ersten Projekten auf einem guten Weg, diese Möglichkeiten zu nutzen und sich den Herausforderungen zu stellen. Nun geht es darum, diese Möglichkeiten auszubauen - Bekämpfung von Lebensmittelbetrug ist z.B. ein prioritäres Thema - und offensiv voranzuschreiten, um das breite Spektrum der virtuellen Welt für eine Stärkung des Verbraucherschutzes zu nutzen. 